\title{
Guest Editorial: Special Issue on Opportunities, Goals and Challenges in Next Generation ICT-Based Systems
}

\author{
Neeli Rashmi Prasad • Albena Mihovska • \\ Rasmus Hjorth Nielsen
}

Published online: 22 August 2012

C) Springer Science+Business Media, LLC. 2012

This special issue has emerged based on the CTIF Annual Workshop (CAW) held in Aalborg, Denmark in May 2011. The CAW has become a recurring event where researchers and scientists from academia and industry present their work within some of the most important research areas. There are many such research challenges still to be addressed and we are happy to present some of the papers doing so. The papers address different areas related to steps towards the Future Internet spanning from the application domain over monitoring, security and privacy to network architectures and optimization.

The first paper is in the application domain and is by Lin Zhang, Yu Liu, Xin Zhan, Xiaomin Yang, Xiaoqiang Chi and Shizhen Zhao. The paper is entitled "Campus View: An Innovative Location and Context-Aware Video Sharing Application on Smart Phone" and presents a view of the future for social network applications utilizing the technologies of videos and smart devices. The case study is conducted on a university and the presented framework augments current solutions such as Google Street View with both real-time videos and usergenerated content. The paper also presents results of the effects of such a solution on device resources. The next paper is "Word Activation Forces: Distinctive Statistics Revealing Word Associations" by Jun Guo, Guang Chen and Weiran Xu. Word activation is an important field within text and speech analysis and an enabler for adding currently unavailable content online. The authors provide an introduction to the field and present an experiment on a data set including more than 10,000 English words.

Within monitoring, security and privacy, the first of three papers is entitled "The Study of Content Security for Mobile Internet" and is authored by Qianfang Xu, Jun Guo and Bo Xiao. This paper presents a detailed framework including subsystems for acquisition, storage,

\footnotetext{
N. R. Prasad · R. H. Nielsen ( $\square)$

Center for TeleInFrastruktur (CTIF), Aalborg University (AAU), Princeton, NJ, USA

e-mail:rhn@es.aau.dk

N. R. Prasad

e-mail: np@es.aau.dk

\section{A. Mihovska}

Center for TeleInFrastruktur (CTIF), Aalborg University (AAU), Aalborg, Denmark

e-mail: albena@es.aau.dk
} 
analysis and processing as well as user and business intelligence. The issues and possible technologies for the individual elements are discussed along with auditing to provide the appropriate security measures. The second paper, entitled "An Empirical Investigation of Filter Attribute Selection Techniques for High-Speed Network Traffic Flow Classification", by Jie Yang, Jing Ma, Gang Cheng, Yixuan Wang, Lun Yuan and Chao Dong investigates different techniques for filter attribute selection. With the vast amount of data already being transferred in current networks, efficient ways of classifying the traffic is essential for both performance and security monitoring. The paper includes detailed results on accuracy of different techniques through the use of real-world traffic. The last paper in this category, "Vulnerabilities of Decentralized Additive Reputation Systems Regarding the Privacy of Individual Votes", is on attacks and defense mechanisms in additive reputation systems and is authored by Antonis Michalas, Tassos Dimitriou, Thanassis Giannetsos, Nikos Komninos and Neeli R. Prasad. Privacy in voting systems is a major challenge to be addressed in order to add trust in many current and future applications. The paper demonstrates the inefficiencies of current protocols providing privacy between users by first surveying the protocols and next categorizing the attacks.

Network architectures and optimization include two papers and the first one by M. V. Ramkumar, Neeli R. Prasad and Ramjee Prasad is entitled "Middleware Architecture for Next Generation Heterogeneous Networks" and addresses converged wired and wireless networks. The paper presents a radio-over-fiber framework and in particular proposes a middleware solution, which includes a number of modules to support heterogeneous technologies. The paper also proposes and evaluates an implementation of a security management module. Optimization of media streaming services is the topic of the paper "QoE Model Based Optimization for Streaming Media Service Considering Equipment and Environment Factors" by Jie Yang, Jing Ma, Gang Cheng, Yixuan Wang, Lun Yuan and Chao Dong. The paper approaches the optimization from a quality-of-experience point of view and includes the capabilities of the equipment along with environmental factors in the allocation of resources. Simulation results of this novel concept are presented, which show how the optimized resource allocation is able to utilize the resources more efficiently and at the same is able to improve the user satisfaction.

In conclusion, we would like to thank all the contributors to this special issue for which we received many excellent papers, but unfortunately could only include a subset. Special appreciation is given to the colleagues at Springer for their continuous efforts in keeping up with the timeline. 


\section{Author Biographies}
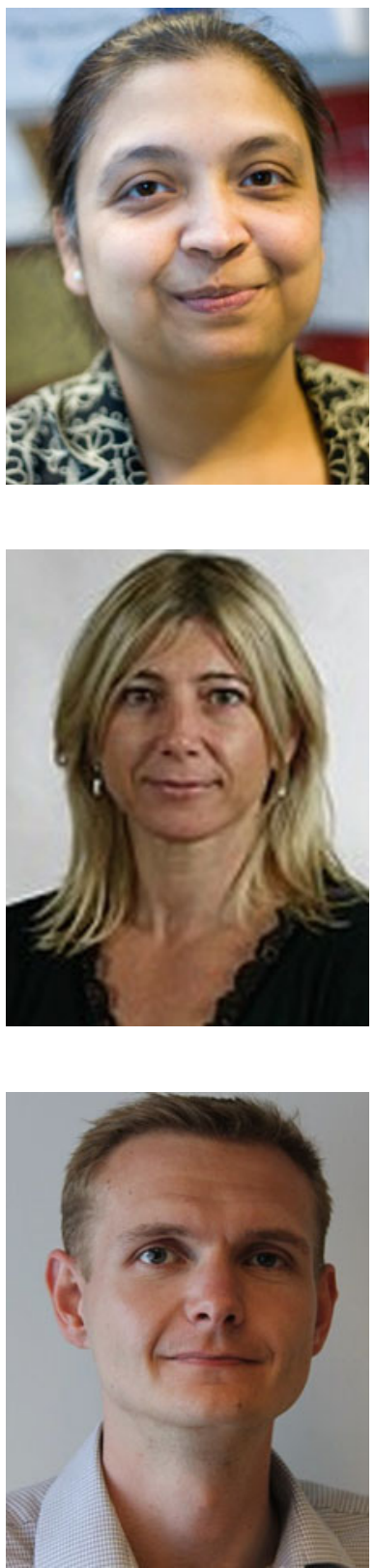

Neeli Rashmi Prasad is an IEEE Senior Member and director of CTIF-USA, Princeton, USA and coordinator of the thematic area "Network without Borders" at Center for TeleInfrastruktur (CTIF), Aalborg University (AAU), Denmark. She is leading the IoT testbed at CTIF Easy Life Lab and the secure cognitive radio network testbed at S-Cogito Lab. She received her Ph.D. from University of Rome "Tor Vergata", Rome, Italy in 2004 and an M.Sc. (Ir.) degree in Electrical Engineering from Delft University of Technology, the Netherlands, in 1997. During her 14 years in industry and academia, she has lead and coordinated several projects in the fields of security, cognitive networking, social networks, IoT, RFID and M2M among others. In the past, she has held multiple positions in the telecom industry including Libertel (now Vodafone NL), Lucent Technologies and T-Mobile.

Albena Mihovska is an Associate Professor at Aalborg University, Department of Electronic Systems. She obtained the Ph.D. from Aalborg University in 2008. She has ten years of experience as a researcher in the area of mobile telecommunication systems. She contributed to the design of a next generation radio communication system through her work as the AAU research team leader in the FP6 EU funded projects WINNER/WINNER II, and CELTIC-funded WIN$\mathrm{NER}+$, with the research laying the foundations for the current LongTerm Evolution (LTE) and LTE-Advanced. She has conducted research activities within the area of advanced RRM, cross-layer optimisation, and spectrum aggregation, the results of which were put forward as IMT-A standardisation proposals to the ITU-R. She has about 86 publications including four books published by Artech House in 2009.

Rasmus Hjorth Nielsen is an Assistant Professor at Center for TeleInFrastruktur (CTIF) at Aalborg University (AAU), Denmark and is currently working as a senior researcher at CTIF-USA, Princeton, USA. He received his M.Sc. and Ph.D. in electrical engineering from Aalborg University in 2005 and 2009 respectively. He has been working on a number of EU and industrial funded projects primarily within the field of next generation networks where his focus is currently security and performance optimization. He has a strong background in operational research and optimization in general and has applied this as a consultant within planning of large-scale networks. His research interests include IoT, M2M, WSNs, virtualization and other topics related to next generation converged wired and wireless networks. 